\title{
L'enseignement de la littérature : un monde à explorer
}

Emmanuel Fraisse

\section{OpenEdition}

1 Journals

Édition électronique

URL : https://journals.openedition.org/ries/2664

DOI : $10.4000 /$ ries.2664

ISSN : 2261-4265

Éditeur

France Education international

\section{Édition imprimée}

Date de publication : 1 décembre 2012

Pagination : 35-45

ISSN : 1254-4590

\section{Référence électronique}




\section{Enseignement et littérature dans le monde}

\section{Introduction}

\section{L'enseignement de la littérature : un monde à explorer}

\section{Emmanuel Fraisse}

Alors que les comparaisons internationales et les démarches d'évaluation sont au cœur de l'approche institutionnelle et économique des systèmes éducatifs dans le monde, les contenus et les modes d'enseignement sont beaucoup plus rarement mis en regard. Depuis plus de trente ans, il existe, à un très haut degré et sur une très large échelle, une remarquable culture comparatiste des systèmes éducatifs, de leur organisation et de leurs performances. Il n'y a guère d'habitude, ni même de discours, de la didactique comparée qui lui soit véritablement symétrique ${ }^{1}$.

Telle est précisément l'ambition de ce dossier de la Revue internationale d'éducation de Sèvres, avec les limites évidentes qui affectent toute démarche d'exploration conduite dans un espace éditorial restreint et un nombre limité d'exemples : contribuer à ouvrir la voie à des comparaisons didactiques entre des pays extrêmement divers par la taille, la situation économique, l'histoire et les cultures éducatives. Et le faire sur un objet d'enseignement universellement partagé, qui est par ailleurs probablement le moins facile à définir de manière stable et indiscutable : la littérature.

\section{UNE DÉMARCHE COMPARATISTE}

Au cœur de l'idée comparatiste, c'est bien la dialectique de la ressemblance et des particularités qui est à l'œuvre. Rien d'étonnant donc à ce que, et au-delà des différences d'échelle, d'histoires et de déterminations sociales, les huit synthèses de situations nationales ici proposées fassent apparaître de nombreuses convergences. Des convergences qui, de la Chine aux États-Unis,

\footnotetext{
1. La plupart des didactiques du curriculum existantes sont centrées sur des objets clairement définis et restreints (technologie, physique, langue), et se limitent, dans leur dimension comparatiste, à des pays proches par la langue ou la culture. Jusqu'ici, la question de la didactique de la littérature est pour l'essentiel restée limitée aux espaces francophones ou bien, mais de manière plus institutionnelle, à l'Europe, ce dont témoigne la bibliographie proposée en fin de dossier par Bernadette Plumelle.
} 
de la Russie au Sénégal, de la France au Danemark, d'Haïti au Québec, sont souvent attendues, mais dont d'autres peuvent constituer en elles-mêmes des surprises pour le lecteur.

C'est notamment le cas de l'importance majeure - ne serait-ce qu'en termes d'horaires - de la littérature et de son enseignement dans tous les systèmes scolaires ici envisagés. On aurait pu imaginer en effet que l'enseignement de la littérature stricto sensu, contenus, exercices et notations, aurait préférentiellement concerné les pays occidentaux, et la France en tout premier lieu. Certes, c'est une des caractéristiques de l'enseignement en Occident que d'avoir longtemps accordé une part importante aux textes littéraires et de les avoir utilisés tout à la fois comme modèles, outil et objet de la formation des élèves. Certes, l'histoire de la France a fait de la littérature et de la langue un fondement de l'idée qu'elle se fait d'elle-même. Mais on verra qu'au Danemark, la question nationale et politique n'est pas moins aiguë qu'en France, tout comme elle peut l'être en Haïti, au Québec ou au Sénégal. Et on relèvera qu'en Chine ou en Russie encore, la littérature constitue l'un des fondements du système de la formation langagière, intellectuelle, civique et morale des élèves. C'est que, de manière presque universelle, la "littérature » est à la fois objet et outil d'éducation et d'enseignement, puisqu'elle est dans le même temps intimement liée aux "valeurs ", à la langue et à son apprentissage et à la maîtrise des discours. Ce que rappelle l'appellation "arts de la langue » en vigueur aux États-Unis, pour la définir comme discipline et objet d'enseignement au sens large. Reste à savoir quelle est cette " littérature » et quelles sont les finalités éducatives et instrumentales qui lui sont conférées selon les pays et les espaces considérés.

Second constat, plus attendu : la relation particulièrement complexe qu'entretient la littérature, saisie comme objet et discipline d'enseignement, avec les «valeurs» et avec l'identité nationale et/ou linguistique. En effet la langue et la littérature (les littératures) qu'elle illustre peuvent être perçues comme héritage légitime et hautement revendiqué, ou au contraire comme conséquence des contraintes de l'histoire. Et, dans tous les cas, chacun a conscience que le «canon» (c'est-à-dire les textes littéraires effectivement retenus comme objets d'étude, d'admiration ou d'imitation) n'est ni naturel ni neutre, pas plus au plan idéologique qu'esthétique.

Troisième constat, qui ne surprendra pas non plus : on relève une hésitation continue entre universel et particulier, comme si la littérature considérée comme objet d'enseignement ne cessait d'être ballottée entre deux pôles : identité et ouverture, vivre entre soi et s'étendre au monde, se connaître soi-même et connaître l'autre. À l'heure de la mondialisation - et la très grande convergence organisationnelle des systèmes éducatifs reposant tous sur la proclamation d'une obligation scolaire longue en est une illustration frappante -, on gardera à l'esprit qu'une des questions posée par l'objet « littérature » est bien celle de la relation entre l'universel et le particulier. C'est évidemment vrai des élèves 
pris individuellement et en groupes, mais également des nations et des cultures éducatives qu'elles ont élaborées ou qui leur ont été léguées par l'histoire. Peut-on un instant imaginer l'horizon d'une littérature authentiquement universelle ? Ou faut-il rappeler que l'universel est toujours pensé à partir de déterminations particulières? Autrement dit : quel est, dans l'enseignement de la littérature, la relation qui s'établit, ici et maintenant, entre le national et le mondial?

\section{LITTÉRATURE ET VALEURS}

À traverser les articles constituant le dossier, on est frappé par le fait que, dans l'ordre de l'enseignement, la littérature se voit confier diverses fonctions, dont une des principales est de contribuer à éduquer les élèves en leur donnant à partager un certain nombre de références porteuses de valeurs. Parmi celles-ci, l'appartenance à une communauté nationale et/ou linguistique.

\section{Enseignement littéraire, État, nation}

Une des principales variations observées dans les différents pays évoqués renvoie tout naturellement au rôle de l'État. Il ne s'agit pas tant ici de l'organisation administrative des systèmes d'éducation - totalement décentralisée dans le cas des États-Unis, très unitaire et déconcentrée dans celui de la France - que de l'intervention des autorités centrales sur les programmes eux-mêmes et de leur attente explicite face à l'enseignement de la littérature. Comme le rappelle M. Martin Guiney, comparant les États-Unis et la France :

L'agence fédérale Department of Education, contrairement au ministère de l'Éducation nationale, ne joue aucun rôle dans l'élaboration des programmes ni dans la définition des finalités et des modalités de l'enseignement; cela est même strictement interdit par la loi.

Inversement, dans un pays aussi vaste et peuplé que la Chine, il y a définition de normes provinciales, elles-mêmes articulées aux normes nationales, et visant à les actualiser. De ce point de vue, le nuancier est sans fin en ce qui concerne l'intervention plus ou moins directe et centralisée des autorités dans ce domaine. Reste que l'opposition bien connue entre systèmes normatifs, très majoritaires dans l'échantillon retenu, et le système américain qui laisse toute latitude au "marché » (les éditeurs de manuels), aux acteurs (les associations de professeurs) et aux autorités locales élues renvoie à une autre opposition: celle qui fixe les fonctions attendues de la littérature comme objet d'enseignement.

Ici encore, les États-Unis continuent à faire figure d'exception, mais on gardera à l'esprit que n'ont été retenus que deux pays en Europe occidentale et un dans le monde anglo-saxon. Selon l'analyse de M. Martin Guiney, et compte 
tenu de la priorité donnée à l'approche des « arts de la langue », c'est de manière très consciente et explicite que la littérature n'est qu'accessoirement définissable par un ensemble de textes de référence susceptibles de donner aux élèves et futurs citoyens américains un appareil de valeurs et de repères idéologiques communs :

La littérature comme matière enseignée signifie donc, en plus de la pratique de la langue, la libération de l'individu par la culture de l'imagination ; la pluralité des voix et des modes d'expression; et le droit à la parole individuelle, plutôt que des techniques d'assimilation d'un corpus hérité de textes.

Peu de pays offrent un tableau aussi différent de celui des États-Unis que le Danemark évoqué par Jørn Boisen. Peut-être, comme le remarque l'auteur, parce que le Danemark, tout comme la Russie ou la France, appartient au « club très select des vieux États-Nations ", mais surtout parce que sa modernisation nationale au cours du XIX ${ }^{\mathrm{e}}$ siècle s'est, pour une large part, opérée sous le patronage de Herder (1744-1803), qui a été l'un des premiers à théoriser la relation indissoluble entre "peuple", "langue " et authenticité culturelle et littéraire ${ }^{2}$. Ce faisant, il s'opposait à l'artificialité du modèle de l'Antiquité classique dont la France avait été le traducteur, l'adaptateur et l'agent de diffusion à travers l'Europe des XVII et XVII ${ }^{e}$ siècles. Autant dire que pour le Danemark d'aujourd'hui, comme pour bien d'autres pays, la littérature et son enseignement restent un lieu de débat idéologique, toujours mouvant mais perpétuellement lisible à travers une ligne d'affrontement constamment prête à resurgir sous de nouvelles formes. De cet affrontement témoigne la mise en place d'un récent "Canon de la littérature danoise » qui entend, contre les séquelles de l'idéologie de 1968, mais aussi contre les excès de la pure "communication ", rétablir fermement les valeurs de l'identité nationale à travers les contours officiels de la littérature à enseigner.

Le cas français est sans doute assez différent. Comme le rappelle Violaine Houdart-Merot, la «francisation » des études littéraires - textes et

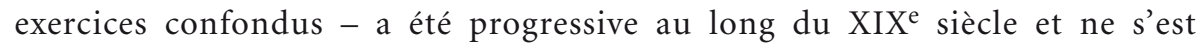
pleinement imposée qu'à partir de la III ${ }^{e}$ République. Reste qu'en France, la relation entre nation et enseignement de la littérature est assurément paradoxale, car ce pays vit encore avec la certitude selon laquelle, tout particulièrement dans l'ordre de la littérature, ce qu'il pouvait avoir de particulier avait justement vocation universelle. C'est vrai de l'Âge classique, qui prétend imiter et surpasser l'Antiquité, c'est vrai aussi du siècle des Lumières, dont le message est à l'évidence à l'usage du monde entier, c'est également vrai des grands textes ou des grands auteurs du XIX ${ }^{\mathrm{e}}$ siècle (Hugo, Zola) et de la première

2. Voir Benedict Anderson, Imagined Communities: Reflections on the Origin and Spread of Nationalism, [Londres, 1983], L'imaginaire national : Réflexions sur l'origine et l'essor du nationalisme, trad. par Pierre-Emmanuel Dauzat, Paris : La Découverte, 1996 ; nouv. éd., coll. « La Découverte poche », 2002. 
moitié du XX $\mathrm{XX}^{\mathrm{e}}$ siècle devenus fondement d'un canon dont les fameux manuels de Lagarde et Michard (1948-1962) ont durablement, et mieux que bien des textes officiels, fixé les contours ${ }^{3}$.

Une disposition intellectuelle analogue est sensible dans la présentation que proposent Lioudmila Troubina et Viktor Chertov de l'enseignement de la littérature en Russie :

La connaissance des œuvres de l'art du langage des peuples d'une Russie multinationale concourt à élargir la représentation qu'ont les élèves de la richesse, de la diversité, du potentiel moral et spirituel de la culture de leur pays et favorise leur prise de conscience de leur appartenance à la culture nationale mais aussi mondiale. La découverte par les élèves russes de la culture des pays étrangers contribue à leur compréhension de l'universalité des valeurs spirituelles, morales et esthétiques et les aide à appréhender la vie et la création d'hommes et de femmes de différents pays.

On notera dans cette perspective que la Fédération de Russie est un des rares États où une possibilité d'étude systématique des littératures étrangères traduites est inscrite dans la scolarité obligatoire, l'objet de cette dimension comparatiste n'étant pas dénué de tout souci national, puisqu'il s'agit de situer la tradition russe dans son rapport aux grands courants thématiques et esthétiques de la littérature universelle.

\section{Langue, littérature, identités}

La question des valeurs d'identité nationale, et de la fonction de la littérature comme expression et fondement de ces valeurs, se pose évidemment sur un mode tout particulier dans les pays que leur histoire a conduits à ne pas hériter d'une littérature exclusivement nationale. C'est le cas des pays issus de la colonisation impérialiste (Sénégal), ou séparés de leur ancienne métropole par une guerre d'indépendance (États-Unis, Haïti) ou les vicissitudes de l'histoire (Québec) et, plus largement, de l'ensemble de l'Amérique, nord et sud confondus, mais aussi des «nouvelles Europes » de l'Océanie. D’une manière générale, tous les pays qui ne relèvent pas de la vieille Europe (et donc du fameux club des vieux États-Nations) et qui ont en partage une langue de scolarisation issue de ces mêmes États-Nations - anglais, espagnol, portugais, français - sont confrontés à l'articulation entre la littérature "nationale » et celle héritée de l'ancienne métropole, du colonisateur. L'affaire est particulièrement enchevêtrée lorsque coexistent deux langues nationales et deux systèmes d'enseignement (Haïti) ou bien une langue officielle et des langues nationales (Sénégal), alors que les textes littéraires nationaux (ou africains, ou de la négritude), pour des raisons

3. André Lagarde et Laurent Michard, Textes et littérature, Les grands auteurs français du programme, Paris : Bordas,

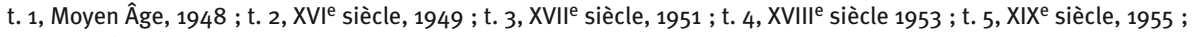
t. 6, XXe siècle, 1962. 
aisément compréhensibles de diffusion et d'édition, liées elles-mêmes aux compétences linguistiques du public lecteur et au prestige et au rayonnement du français sont écrits et publiés dans cette langue ${ }^{4}$.

Quel équilibre adopter au sein d'une même langue entre textes «nationaux » et textes issus de l'ancienne métropole? La réponse donnée dans la plupart des cas observés est d'opérer un découpage temporel : les textes «nationaux » viennent illustrer la littérature contemporaine, qui presque toujours coïncide avec l'Indépendance, la littérature ayant été dans bien des cas tout à la fois un des ferments et une des preuves de cette indépendance ${ }^{5}$. Or, contrairement à l'ordre chronologique qui a prévalu en France jusqu'aux années 1970 (et qui laissait supposer un progrès constant), la littérature dans le monde n'est plus exclusivement étudiée du passé vers le contemporain et selon le découpage par siècles cher au Lagarde et Michard évoqué plus haut. On ne s'étonnera donc pas de voir qu'au Québec, selon l'enquête conduite par Olivier Dezutter, Julie Babin, Marcel Goulet et Lise Maisonneuve, deux ouvrages sur trois étudiés ou lus dans le cadre scolaire du secondaire francophone sont québécois. Inversement, au Collège (deux années entre le secondaire et l'université), les textes de référence du passé relèvent massivement du patrimoine "français » au sens large : Molière, Voltaire, Hugo, Baudelaire, seul le contemporain venant illustrer la littérature québécoise proprement dite. On observe une tendance analogue aux États-Unis, avec cette réserve que seuls les élèves de la classe terminale du secondaire (en dehors donc de la scolarité obligatoire) sont confrontés à la "littérature britannique ", c'est-à-dire à la littérature en anglais antérieure au XIX ${ }^{\mathrm{e}}$ siècle. Mais, comme le suggère M. Martin Guiney, quoique la présence de textes littéraires dans l'enseignement américain soit très accessoirement à vocation «identitaire », on peut imaginer que ces textes du passé sont assimilables au patrimoine national, les États-Unis ayant en quelque sorte accompli la promesse anglaise, comme le Nouveau Testament a accompli l'Ancien.

Dans le cas d'Haïti, Darline Alexis montre la complexité de l'articulation entre littérature "nationale» et littérature française. Certes, les programmes et les manuels de référence renvoyant aux deux littératures française et haïtienne (Lagarde et Michard d'un côté, Berrou et Pompilus de l'autre) semblent se répondre harmonieusement. Mais l'auteure de l'article tend à montrer qu'en définitive, c'est la même «idée » de la littérature qui prévaut : celle d'une approche réductible à l'histoire littéraire, alors que les réformes en cours en Haïti mettent l'accent sur un élargissement des corpus à la littérature africaine et haïtienne dans le cadre d'une organisation par séquences thématiques, selon les pratiques actuelles de l'enseignement secondaire français.

4. Voir Pascale Casanova, La République mondiale des lettres, Paris : Seuil, 1999 ; nouv. éd., 2008, coll. " Points essais $"$.

5. Bernard Mouralis, Littérature et développement. Essai sur le statut, la fonction et la représentation de la littérature négro-africaine d'expression française. Thèse pour le doctorat, Université de Lille 3, 1978, Lille, ART, 1981 ; nouv. éd., Paris, Silex, 1984. 


\section{Qu'est-ce que la littérature?}

"Qu'est-ce que la littérature? » À cette question inévitable, dont Sartre après beaucoup d'autres avait bien compris qu'elle était sans réponse satisfaisante ${ }^{6}$, Roland Barthes a répondu par une semi-boutade aux lendemains de 1968 : « la littérature, c'est ce qui s'enseigne, un point c'est tout ${ }^{7}$. " C'était en 1969, lors d'un colloque consacré à l'enseignement de la littérature dans une communication où, avec les meilleurs esprits de son temps, Barthes mettait en cause l'idéologie de l'histoire littéraire dont le Lagarde et Michard semblait le symptôme et l'agent le plus visible. Mais, d'un tout autre point de vue, et c'est ce que nous aident à comprendre les articles de ce dossier, c'est justement le travail de normalisation institutionnelle et de définition par la liste (le canon) que l'École impose très généralement, voire suggère à travers ses outils et ses exercices, qui permet à tous les acteurs d'avoir une idée de ce qu'est «la littérature ». Dans ces conditions, on s'accordera à estimer que, pour une part, la littérature est bien ce qui s'enseigne comme telle.

De ce point de vue, l'analyse proposée par Chen Wei et Li Qin de la notion de yuwen rapportée à l'enseignement et signifiant, tout à la fois « langue ", "écriture ", « littérature » et «culture » est particulièrement éclairante. Non seulement pour la Chine, où les Analectes de Confucius font partie de la « littérature " au même titre que les romans de $\mathrm{Pa}$ Kin, mais pour le monde entier. En effet, la notion de "littérature » est en réalité fort récente ${ }^{8}$, y compris en Occident, et il n'est pas certain que sa définition soit unanimement partagée. À l’Âge classique, "littérature » signifiait « connaissances », "doctrine ${ }^{9}$ ». L'article de Violaine Houdart-Merot rappelle avec force que les «Belles-lettres » ellesmêmes n'ont pas toujours été réduites, comme on le croit trop souvent aujourd'hui, à la fiction ou aux trois genres devenus canoniques : théâtre, poésie, roman. D'ailleurs nul ne viendrait s'insurger en voyant les Essais de Montaigne classés comme "littérature ", alors qu'on peut tout aussi bien les considérer comme relevant de la philosophie. Il en va de même pour Michelet et beaucoup d'historiens d'hier. Ce qui est ici en question, c'est bien une interrogation sur la « littérarité " chère à Roman Jakobson (1896-1982), et dont plusieurs articles du dossier (Chine, États-Unis, France) se font l'écho : existe-t-il un indiscutable critère permettant d'accepter ou de rejeter une ouvre comme "littéraire » ou «non littéraire» selon les cas? Faute de répondre au fond de la question (il

6. Jean-Paul Sartre, Qu'est-ce que la littérature ?, [Les Temps modernes, 1947] in : Situations II, Paris : Gallimard, 1948 ; nouv. éd., coll. « Folio Essais », 1992.

7. Roland Barthes, « Réflexions sur un manuel », in : Serge Doubrovsky et Tzvetan Todorov (dir.), L'Enseignement de la littérature, Paris, Plon, 1971, p. 170-177.

8. Voir Philippe Caron, Aux origines de la notion contemporaine de "Littérature ». Le Lexique et la configuration idéologique des grands secteurs du savoir profane en langue française de 1680 à 1760, Thèse pour le doctorat, Université de Nancy 2, 1987.

9. Première édition du Dictionnaire de l’Académie (1694) : « Toute sorte de science et de doctrine. [...] Grande littérature, profonde littérature. » 
existe à l'évidence des degrés, des régimes de "littérarité » plus ou moins intenses selon les œuvres), force est de reconnaître que c'est bien l'appréciation historique et sociale, à tel moment et en fonction de tel public, qui est à même d'aider sinon à définir la littérature, du moins à en cerner les contours. Ce que précisément fait l'institution scolaire au sens large.

D'où un paradoxe fondateur: la naissance de la "littérature », dans l'acception courante d'œuvres de l'esprit fondées sur leur qualité d'expression en langue nationale, leur capacité à produire de l'émotion chez le récepteur et leur contenus formateurs, est indissociable de son enseignement même. Plus, on peut dire qu'au sens moderne du terme, l'enseignement de la littérature, dont la vocation est universelle, est indissociable du cadre national : c'est l'État-Nation qui est à l'origine de l'enseignement littéraire en langue nationale (ou en langue partagée) et qui, en retour, assure sa perpétuation ${ }^{10}$.

\section{LITTÉRATURE ET ÉCOLE : PRATIQUES OU CONNAISSANCES?}

Au-delà des «valeurs », le dossier a pour ambition de mettre en lumière les variations des pratiques réelles des élèves au long de la scolarité. Or si la question des valeurs, pour lesquelles, dans la plupart des articles, l'accent est mis sur le second degré, voire le second degré long, est très bien partagée, celle des pratiques est à la fois moins convergente et moins étudiée dans le détail. Sans doute parce que les limites proposées interdisaient des analyses de terrain ; mais également parce qu'il est infiniment difficile de rendre compte de la réalité de l'enseignement, ne serait-ce qu'à cause de la variété des enseignants et des modes de transmission. C'est donc sur l'esprit de certains exercices et sur l'impact de ceux-ci sur les modes d'examen qu'on s'attardera.

\section{Récitation et poésie}

Dans l'ordre des pratiques liées aux genres littéraires, il apparaît que la poésie est très largement présente dans les petites classes de l'école élémentaire en Russie, en Chine, en Haïti, en France ou au Sénégal. Dans tous les cas, la récitation, fondée à la fois sur la mémorisation, l'imitation et l'oralisation, s'impose comme le premier mode d'accès aux textes littéraire et de familiarité, voire d'intimité avec eux. On notera qu'en Chine, la mémorisation et la récitation concernent très souvent des textes anciens dont la compréhension est souvent malaisée. La situation n'est pas très différente en vérité quand un écolier français

10. Ce qui explique également, à la fin du XIXe siècle et au début du XXe siècle, la naissance en Europe d'une véritable littérature de romans scolaires à destination des élèves du primaire, et donc issus des classes populaires. Voir Patrick Cabanel, Le Tour de la nation par des enfants. Romans scolaires et espaces nationauX (XIXe-XXe siècles), Paris : Belin, 2007. 
récite une fable de La Fontaine. En proposant un regard historique sur les soubassements de l'école coloniale et sa transformation à la suite de l'Indépendance et en consacrant une part importante de son analyse à l'école élémentaire au Sénégal, Abdoulaye Elimane Kane montre à quel point la récitation peut être un exercice structurant, et l'entrée la plus naturelle au cœur de la parole poétique. La grande tradition senghorienne de reviviscence de la parole par la poésie et le conte, celle dont l'Anthologie de la nouvelle poésie nègre et malgache d'expression française (1948) avait marqué la naissance, est bien à l'œuvre dans les manuels qui ont succédé au célèbre Mamadou et Bineta de l'époque coloniale.

\section{Exercices et examens}

Reste que, dans leur majorité, les contributions s'attardent plus sur les connaissances culturelles à acquérir que sur la nature des exercices et des compétences attendues des élèves. Comme le remarque Violaine Houdart-Merot, la pratique de la littérature à l'école reste, en France, plus une affaire de lecture que d'écriture, de réception et de commentaires que de production, de connaissances que de pratiques.

À vrai dire, on a bien l'impression que ce phénomène n'est pas purement français, dans la mesure où la dimension créative de l'approche des textes littéraires va à l'encontre des normes d'évaluation les plus répandues et les plus simples à expliciter et à mettre en ouvre ${ }^{11}$. On touche ici à une dimension fondamentale, qui mériterait à elle seule une étude approfondie : celle des " cultures scolaires ${ }^{12}$ ", qui sont loin de se résumer aux valeurs ou aux références mais qui impliquent des comportements et des manières de faire très souvent intériorisés. Il y a déjà un écart entre le "canon » et les manuels; un autre écart entre les manuels ou outils de référence et les textes réellement vus par les élèves. Il y a un écart plus grand encore la réception souhaitée par les professeurs et les productions attendues des élèves. Or, à ce stade, on ne peut que relever la force et la prégnance des examens qui, à proprement parler, modèlent les comportements de l'ensemble des acteurs concernés : professeurs et élèves, et bien souvent leurs parents. Certes, on ne sera pas surpris de voir les États-Unis ou le Danemark relever d'une culture éducative moins contraignante que la France ou la Chine. Reste que, pour la plupart des cas étudiés, la dimension de l'examen - qu'il s'agisse de l'examen de fin d'études ou plus généralement du concours d'entrée à l'université - joue un rôle décisif, qui conduit à une maîtrise très codée d'exercices répétitifs (c'est le cas en Chine), dont la réussite conditionne l'avenir des élèves.

11. La dissertation « à la française » est devenue beaucoup plus exigeante aujourd'hui qu'il y a quarante ans. C'est aussi le cas de la composition de philosophie au baccalauréat, dont le système d'appréciation et de notation est régulièrement mis en cause, et dont la sévérité et l'obscurité contribuent à expliquer la crise de vocation dans les filières littéraires.

12. L'expression a été très largement diffusée à la suite des travaux d'André Chervel. Voir notamment La culture scolaire. Une approche historique, Paris : Belin, 1998. 
Et chacun sait qu'il est infiniment plus facile de maittriser un code (celui de l'exercice, souvent fractionné, ne serait-ce que pour des raisons docimologiques évidentes) que de mettre en œuvre des savoirs complexes qui impliquent non seulement la maîtrise de la langue et de dispositifs discursifs ou rhétoriques, mais l'expression d'une sensibilité et une appréciation esthétique qui engagent l'individu. Il est naturellement plus facile de vérifier des connaissances d'histoire littéraire au moyen d'un questionnaire ou de son équivalent, de noter la correction orthographique, morphologique ou syntaxique d'un exercice de grammaire que d'apprécier une production "littéraire " (de critique ou de création), où un élève engage sa sensibilité, voire sa personnalité. Pour le dire crûment : il est plus facile pour tous les acteurs de la chaîne éducative de vérifier des connaissances ponctuelles, où la mémorisation et l'imitation jouent un rôle majeur, que d'évaluer des compétences en vraie grandeur.

\section{ENSEIGNER LES HUMANITÉS DANS LE MONDE D'AUJOURD'HUI}

Pour être jugé fondamental, l'enseignement de la langue / littérature n'est pas moins entré en crise, et ce à l'échelle du globe. Nombre des articles s'en font l'écho, des États-Unis à la Chine, de la France à la Russie. La menace sur les études littéraires à l'école prend deux visages : celui de la concurrence de la "communication» et de l'utilitarisme ${ }^{13}$, voire du «cynisme" évoqué par Lioudmila Troubina et Viktor Tchertov; celui d'une relative inadaptation de ces études, et du danger que les élèves ne soient conduits à les déserter.

Dans le débat en cours, à travers le monde entier, la question posée est bien tout à la fois celle du périmètre et des objectifs des études "littéraires" - ou des "humanités» au sens large - et, en filigrane, celle de leur utilité et de leur avenir. S'il est clair que le modèle d'hier - celui de la formation d'élites restreintes de lettrés, d'érudits et d'orateurs - n'a plus de sens, et si celui d'aujourd'hui, fondé sur la seule étude de textes "littéraires" (de préférence nationaux), est en crise, d'autres directions sont imaginables pour l'enseignement, et notamment l'enseignement secondaire. Parmi elles, une orientation acceptant résolument de dépasser le cadre étroitement « littéraire » des études humanistes, faisant une large place aux sciences sociales et à la culture générale, et acceptant de fondre le littéraire dans le culturel, en étendant par exemple la "littérature " à l'image, comme c'est déjà le cas aux États-Unis. Si une telle évolution n'est pas provoquée et encouragée, on risque fort de voir, à l'intérieur même des enseignements des langues nationales, triompher des enseignements à dimension essentiellement communicative. Dans l'ordre de l'apprentissage des langues

13. Pour une dénonciation aux accents pamphlétaires de cet utilitarisme aux États-Unis, voir Martha C. Nussbaum, Not for Profit. Why Democracy needs Humanities, Princeton and Cambridge : Princeton University Press, 2010. 
étrangères, ces modèles ont déjà largement remporté la partie : reste à se demander, au-delà de leur efficacité instrumentale, leur réel impact en termes de formation humaine et culturelle.

Quelle que soit la définition qu'on donne des humanités - et elle ne peut être que mouvante -, leur enseignement se doit en définitive d'explorer deux directions principales.

La première concerne la notion de littérature mondiale, et la place des littératures non occidentales. Problème redoutable qui renvoie sans cesse à la question du point de vue adopté et des instances de légitimation. Et qui suppose une réflexion sur les langues et la traduction, saisie comme expression des rapports nécessairement tendus et conflictuels entre langues et cultures dans le monde. À cet égard, il suffit de s'interroger un instant sur le Prix Nobel ${ }^{14}$. Plus ouvert aux femmes et au Sud depuis deux décennies (est-il besoin de rappeler qu'un écrivain de langue arabe - Naguib Mahfouz en 1988 - et qu'un Chinois - Mo Yan en 2012 - ont été lauréats ?), le Prix Nobel reste un prix occidental, et à coup sûr un prix des langues occidentales : aucun écrivain non traduit dans plusieurs des «grandes " langues de l'Occident ne peut parvenir à la notoriété mondiale ${ }^{15}$. Sur un plan plus général, la place des «autres », est bien au cœur de la rénovation, ou plutôt de la continuité, des humanités. Pour prendre un exemple particulier, mais dont on voit que son principe est extensible à de nombreuses autres situations nationales: peut-on un instant imaginer que l'enseignement de littérature «française» en France ne s'étende pas à la francophonie?

La seconde direction de réflexion, plus radicale encore, renvoie à la notion de culture elle-même : peut-on aujourd'hui dissocier langue / littérature / culture ? Bien évidemment, non. Or le système universitaire et secondaire français, pour ne parler que de lui, ne cesse de diviser les approches de ces phénomènes en une multitude de spécialités ou de disciplines trop souvent étanches.

On rappellera enfin qu'au cœur des humanités réside une dimension fondamentale, qui est véritablement digne d'être universelle : celle du doute et de l'interprétation. Si un mot donc pouvait en effet définir et justifier les humanités, c'est que, par nature et fonction, comme disaient les grammaires scolaires d'hier, elle ne peuvent être que critiques. Au sens plein du terme.

14. Voir James F. English, The Economy of Prestige: Prizes, Awards, and the Circulation of Cultural Value, Cambridge : Harvard University Press, 2008.

15. Voir Pascale Casanova, La République mondiale des lettres, op. cit. 
